\title{
Case Study Application of Business Intelligence in Digital Advertising
}

Dželila Mehanović, International Burch University, Bosnia and Herzegovina*

Nermina Durmić, International Burch University, Bosnia and Herzegovina

\begin{abstract}
This paper demonstrates the application of business intelligence in decision making in digital advertising through a case study. Data used for analysis was collected during a test phase of an advertising platform. The study analyzes multiple types of traffic related to countries, browsers, household incomes, and days of a week. Beside tabular reports, the paper presents how to visualize those results using Python libraries to make them more visually appealing. Furthermore, logistic regression was used to build models to detect relationships between the number of impressions and clicks. Finally, the authors propose multiple combinations of data that could be used to create different reports that lead to smarter decision making and cost effectiveness.
\end{abstract}

\section{KEYWORDS}

Business Intelligence, Campaigns, Data Analysis, Linear Regression, Marketing, Python, Reporting, Traffic

\section{INTRODUCTION}

The term digital advertising denotes all forms of advertising over the Internet to reach an audience and generate traffic. AT\&T released the first banner ad in 1994 which is considered as the beginning of the development of digital advertising (WIRED Staff, 2010). Fuxman et al. (2018) state that digital advertising achieved higher spending than traditional advertising for the first time in 2013. After that, the budget spent on digital advertising has been increasing and it is expected to reach $\$ 375$ billion until the end of 2021 (Dautovic, 2019).

According to the statistics in Dautovic (2019), the number of devices that have active ad blockers increased to 615 million in 2019, which is 473 million more than one year before in 2018. Moreover, it is reported that $76 \%$ of advertisers do not use behavioral data to target the audience. Improper targeting could be the reason why people use ad blockers. This means that people are overcrowded with ads they are not interested in.

Different groups of authors wrote about the essential role of business intelligence in digital advertising (Kursan \& Mihić (2010), Kurniawan et al. (2014), Verma (2018)). Business intelligence (BI) refers to technologies and processes related to data collection, preparation, data analysis, and visualization. The goal of BI is to help organizations in the process of making final decisions that are considered intelligent and factual. Using BI, organizations can make predictions, analyze performance 
and customer behavior, and find ways to increase profit. BI supports data analysis, querying, reporting, and visualization. (Tableau, 2020).

Bhosale et al. (2020) described the role of business intelligence in digital marketing. They concluded that real-time execution in the domain of marketing increases the need for the application of the business intelligence and the knowledge of how to apply it. The authors of this study managed to find plenty of works related to the business intelligence understanding, but without a detailed description of the applied methods and reports that could be used. In response to the identified lack of literature, the aim of this study is to describe the most common methods of business intelligence application in digital marketing in detail and elaborate the resulting reports obtained through those methods.

More specifically, the purpose of this paper is to analyze which types of campaigns and targeting options bring the most audience to digital advertising. To accomplish this goal, the aim of this paper is to see how targeting options could be adjusted when displaying ads to users so that people see personalized ads. Furthermore, this paper aims to help companies to use the advantages of digital marketing without unnecessary loss of money and time. In this paper, the authors present how business intelligence helps businesses to make smart decisions in digital advertising and not to lose extra resources through a case study of tribeOS advertising platform.

\section{BACKGROUND AND LITERATURE REVIEW}

This section presents the meaning and definition of digital advertising and business intelligence fields separately, and ways they can work together, through an overview of relevant literature.

\section{Digital Advertising}

Traditional marketing involves newspapers, TV, and magazines used to reach the local population. This kind of advertising sends one message to the entire audience, without any targeting including the application of filtering and personalization. On the other hand, digital advertising refers to the advertising over web, through email and mobile phones. It is globally oriented and based on filtering and personalization (Greenspan, 2018).

In traditional marketing, there are one-time payments and success is measured by inspecting whether the sales had increased during a given period. In digital advertising, payments occur all the time. Success is measured by the number of impressions, clicks, and sales. Impressions are obtained every time an ad is displayed on a user screen and used for building brand recognition. Cost per thousand impressions or Cost Per Mile (CPM) is used as one of the payment options in digital advertising (Ecommerce Answers, 2020). Beside CPM, there is a Cost Per Click (CPC) option, where a certain amount of money is paid for each click (Ecommerce Answers, 2020). The third option is based on the number of sales, which is Cost Per Sale where advertisers pay for each sale or conversion generated by the ad.

In contrast to traditional advertising, digital advertising provides support for different campaign targeting options to reach the right customers. To achieve this, there are multiple segmentation criteria for targeting the audience such as demographics features like age, gender, education; geographic location such as country, region, city, zip code; and behavioral values such as spending habits (Yesbeck, 2018). Generally, there are two types of targeting: contextual and behavioral. Contextual targeting is done based on keywords, topics, language, and location, while behavioral targeting is related to the browser history, sites visited, time spent on those sites, and recently searched items (Zhang \& Katona, 2012).

\section{Business Intelligence}

Business intelligence refers to the infrastructure responsible for collecting, storing, and interpreting data generated from business operations. It includes a variety of processes such as analytics, data 
mining, data warehousing, benchmarking, result forecasting, and reporting. BI provides easy to interpret reports using generated data. These reports are used by management to make smart business decisions (Frankenfield, 2019). Data analysis and reporting refer to the process of data analysis using different tools and building reports that are shared over the Web (Imanuel, 2020). Data warehousing is used to integrate and manage data from different sources (Chaudhuri \& Dayal, 1997). Performance management is used to increase performance and enhance decision-making (Jalloh \& Ming, 2020).

The need for business intelligence is derived from the fact that managers tend to make bad decisions when they have incomplete or incorrect information. This is a problem that business intelligence tends to solve by analyzing data that is ideally presented using dashboard metrics, which provides complete and accurate information to management in order to make better decisions (Waldron, 1997).

Business intelligence is adopted and applied in many different areas of business. It found its place in finance, sales, marketing, customer service, human resources manufacturing, and others (Sharda et al., 2014). In marketing, BI tracks campaign returns and helps to adjust spending to achieve a better return on investment (ROI) (Zamfir et al., 2016), used to measure investment's profitability. BI is used to specify objectives, evaluate business performance against those objectives, and identify opportunities and weaknesses, which altogether leads to better decisions about future business activities.

In marketing, it is important to identify customer needs and value to enable decision-making about the audience targeting. Furthermore, it is important to track campaign effectiveness to detect opportunities for cost savings and revenue increments. To track campaign effectiveness, we can use $\mathrm{BI}$ to collect data and create dashboards and metrics to measure and monitor performance (Sharda et al., 2014).

\section{Business Intelligence Applications in Digital Advertising}

With the aim of applying business intelligence in digital advertising, authors of Kurnia \& Suharjito (2018) collected data from social media such as Facebook and Twitter to create a dashboard using business intelligence and monitor the performance of news-related posts. They identified the topics of interest using machine learning techniques such as Naive Bayes, SVM, and Decision Tree, where SVM achieved the best accuracy of $78.99 \%$. Furthermore, they made a data warehouse to perform calculations and summarizations alone without the need for manual operations. Research about campaign success (Nichols, 2013) presented the analysis of a client case that advertised through TV, radio, and print media. Data analysis discovered that TV advertising used up $75 \%$ of the budget for one campaign, while YouTube ads used up $6 \%$ of the budget and were two times more effective in leading to sales.

According to Bhosale et al. (2020), the main objectives of business intelligence applications in digital marketing are researching and identifying the right data and following the ROI status. Metrics of evaluation need to be defined and business intelligence should be used to build dashboards where all relevant details are presented in a clear manner that executives could use later on to make informed conclusions. Furthermore, Kurniawan et al. (2014) posed the most important questions in the field of marketing for which business intelligence seems to be the right tool to find answers. To do digital advertising efficiently, it is necessary to identify how to get valid information about customers. Also, the information should be obtained quickly. Once data is collected, we must know how to interpret it and make decisions based on the presented information.

Business intelligence provides support for fact-based decisions rather than using unfounded assumptions. Tilak (2020) stated that business intelligence in marketing helps identifying customer needs and values which is helpful when designing campaigns. She lists several advantages of applying business intelligence in marketing, such as quality and generating real-time reports, which helps to analyze customer behavior and define customized targeting. Chaudhuri \& Dayal (1997) proposed Bayesian predictive analysis to optimize advertising and build a predictive model. This kind of analysis has several advantages such as data borrowing, updating, augmentation, and hierarchical 
modeling. Besides that, the knowledge about customer behavior and location-related data enable more accurate predictive models.

\section{RESEARCH METHODOLOGY}

\section{Dataset}

The dataset used in this research contains the traffic collected from the advertisement platform tribeOS (tribeOS.io, 2020). tribeOS was established in 2019 to provide high-quality traffic and fraud protection for ads. Data was collected in real-time by displaying ads to users and tracking generated impressions, clicks, and sales. This platform enables interaction between advertisers and publishers. Advertisers created campaigns and adjusted targeting options. Data was stored in MongoDB (Singh \& Sharma, 2017) which contains separate collections of traffic for each targeting group. These targeting groups are available to advertisers for adjustment when creating campaigns. Available targeting groups are: distribution channel (app or site), demographic targeting including age and gender, targeting by device type, household income, browsers, operating systems, mobile device types, languages, locations, topics, keywords, apps, and sites. There is also an option of scheduling targeting for specific days or times of day. Table 1 lists all collections that authors took from this system for analysis.

All collections have several common fields such as campaign ID, advertiser ID, impressions, clicks, sales (keeps the amount of money generated by sale), and the number of sales which counts number of sales, timestamp, and data related to that campaign. Values for campaign and advertiser IDs are kept in other collections that do not contain traffic data. Beside these fields, every collection contains traffic columns specific for a given collection with the name and ID of traffic objects. Collection with country traffic contains fields such as country name, country code, and country ID. The same pattern was used for the rest of the traffic data. The data type of the object is a string and IDs are integer values.

\section{Data Analysis Methods}

Python (Zhang, 2015) was used for data analysis because it is recognized as the fastest-growing programming language by many researchers and it is a good choice for data analysis due to its flexibility and stable libraries (Nagpal \& Gabrani, 2019). More precisely, the authors used pandas (Pandas, n.d.) which is a Python library for data analysis. Beside Python, authors used Mongo database queries for some parts since authors have access to MongoDB where all data is stored.

Linear regression is the most common type used in predictive analysis. It models relationships between variables (Yale, n.d.). There is a simple and multiple linear regression. Simple linear regression has one explanatory and one dependent variable, while multiple regression has multiple explanatory variables and one dependent variable (Statistics Solutions, n.d.). The following equation (1) presents simple linear regression where $\mathrm{y}$ is a dependent variable and $\mathrm{x}$ is an explanatory or independent variable. The $\mathrm{b} 0$ and $\mathrm{b} 1$ are coefficients.

$y=b 0+b 1 x$

\section{DATA ANALYSIS}

\section{Data Preparation}

During the data analysis process, the authors didn't use all fields available in the dataset. The traffic object name, number of impressions, clicks, and sales were the fields of the main interest. Also, it 
Table 1. Dataset collections

\begin{tabular}{|c|c|c|}
\hline Collection name & Date range & Number of instances \\
\hline Browsers & $\begin{array}{l}2019-10-09 \\
2020-08-19\end{array}$ & 13027 \\
\hline Device types & $\begin{array}{l}2019-10-09 \\
2020-08-19\end{array}$ & 2837 \\
\hline Device brands & $\begin{array}{l}2020-04-19 \\
2020-08-19\end{array}$ & 3855 \\
\hline Device models & $\begin{array}{l}2020-04-19 \\
2020-08-19\end{array}$ & 12363 \\
\hline Operating systems & $\begin{array}{l}2020-02-01 \\
2020-08-19\end{array}$ & 3709 \\
\hline Household income & $\begin{array}{l}2020-04-22 \\
2020-08-19\end{array}$ & 2604 \\
\hline Countries & $\begin{array}{l}2019-10-09 \\
2020-08-19\end{array}$ & 4374 \\
\hline Regions & $\begin{array}{l}2020-02-05 \\
2020-08-19\end{array}$ & 33449 \\
\hline Cities & $\begin{array}{l}2020-04-08 \\
2020-08-19\end{array}$ & 208556 \\
\hline Zip codes & $\begin{array}{l}2020-03-16 \\
2020-08-19\end{array}$ & 236443 \\
\hline Time days & $\begin{array}{l}2020-04-01 \\
2020-08-19\end{array}$ & 774 \\
\hline Time hours & $\begin{array}{l}2020-04-20 \\
2020-08-19\end{array}$ & 5767 \\
\hline Placements & $\begin{array}{l}2019-12-01 \\
2020-08-19\end{array}$ & 53681 \\
\hline Placement categories & $\begin{array}{l}2020-04-26 \\
2020-08-19\end{array}$ & 741 \\
\hline
\end{tabular}

was possible to group data by specific campaign or advertiser. Data fields such as year, month, week, and different amount fields were excluded from the dataset for further analysis.

Although traffic names such as country name, browser name, device type name, and others are string values, they are normalized across the dataset. Beside the name, there is also the ID value. This is because there is another collection for each of these dictionaries with a defined set of values, which were retrieved and stored in these collections.

The date when traffic was captured was expressed using the Unix timestamp. It was converted to a human-readable date to generate reports and dashboards. After this part of dataset analysis, the authors found that there was no need for large data normalization. Below is an example of one document from a collection related to the country's traffic. This document contains fields selected by the authors to use to propose several reports that could be tracked to increase the campaign performance.

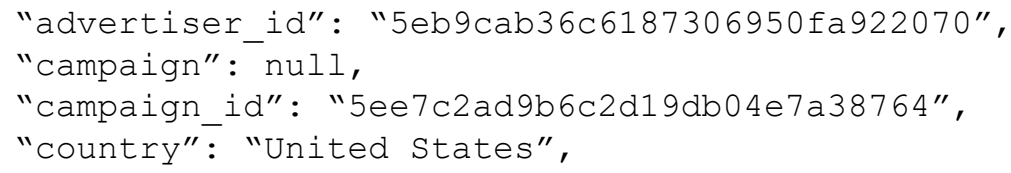




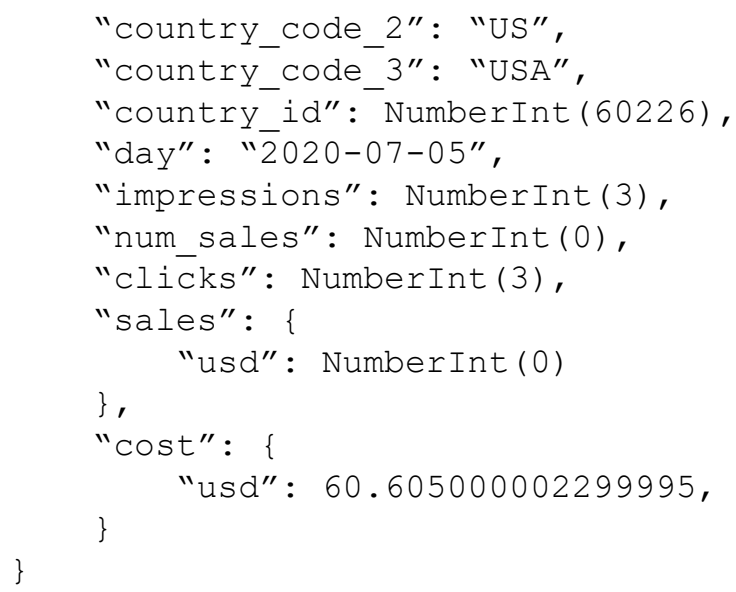

\section{Data Analysis}

This section presents the analysis that was done before creating reports. The analysis was performed using the dataset described in the previous section. The authors selected this dataset because it contains the real traffic data collected through an advertisement network. Although this data was sufficient for this paper, it is important to mention that the dataset does not contain high-quality traffic because it was generated during the testing phase of the network. Results obtained through the steps described in this section are presented in the next section.

Generally, available collections can be grouped in several categories: locations, time, user-agent related items, and placements. For the purpose of this study, the authors analyzed only one type from the group.

The analysis starts with the countries collection to find from how many countries traffic was generated and to see which are the top countries. This was done using pandas function unique() and aggregation.

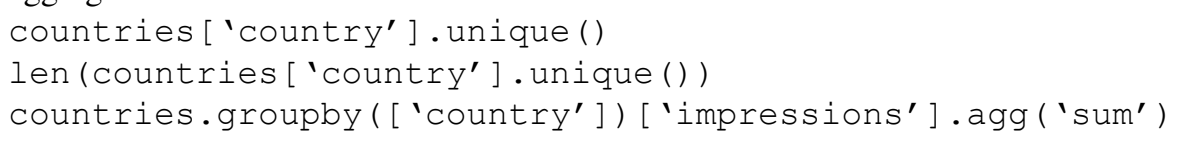

Beside tabular data representation, folium (Folium, n.d.) Python library was used for geospatial data analysis to visualize data on a map. Below is a block of code used to generate the map. The data frame world map was used containing country name, latitude, longitude, and the total number of sales for a specific country.

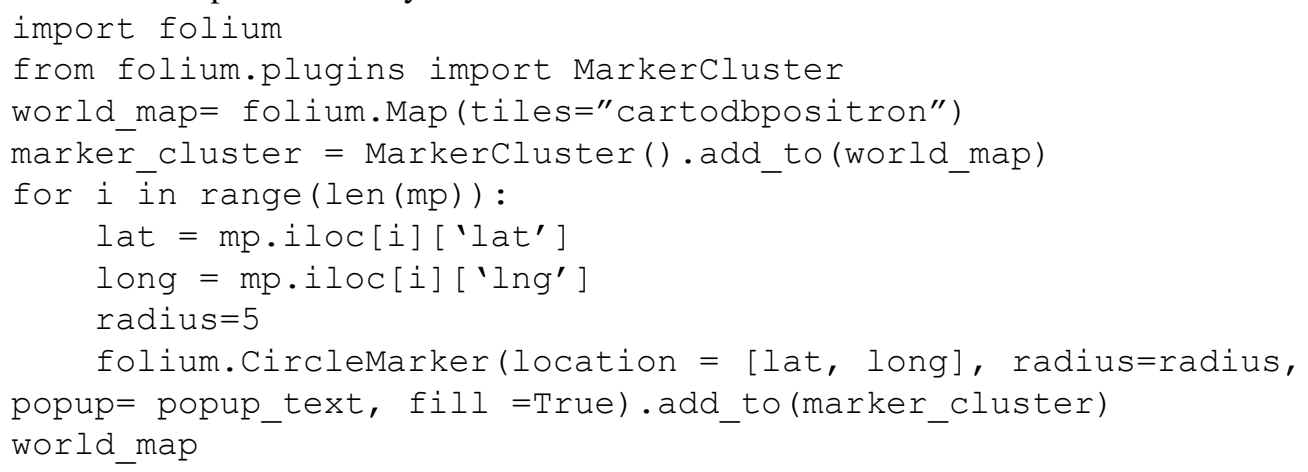

Similar steps can be performed for reporting related to other location types such as regions, cities, and zip codes.

Next, the distribution of impressions was found, together with clicks and sales per day. The authors used similar query to find group data by day of the week. 
days.groupby ( [ 'day_of_week'] ) [ 'impressions' ] .agg ( 'sum' )

Later, matplotlib (Matplotlib, n.d.) python library was used to visualize data in the form of bar charts. The block of code used to create charts is given below.

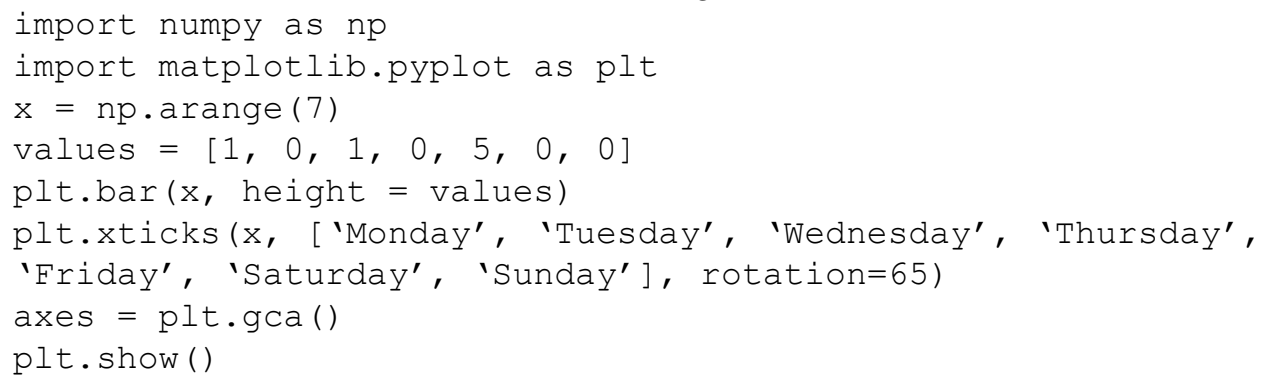

The same steps can be performed to obtain reports by hours. Based on the result, we can decide what time of day has the highest traffic and adjust targeting accordingly.

Browsers, operating systems, device types, models, and brands are detected by user-agents (Kline et al., 2017). The authors analyzed the traffic of browsers presented below, in order to define those with the highest number of impressions, clicks, and sales. After that, pie charts were built using the given data frame.

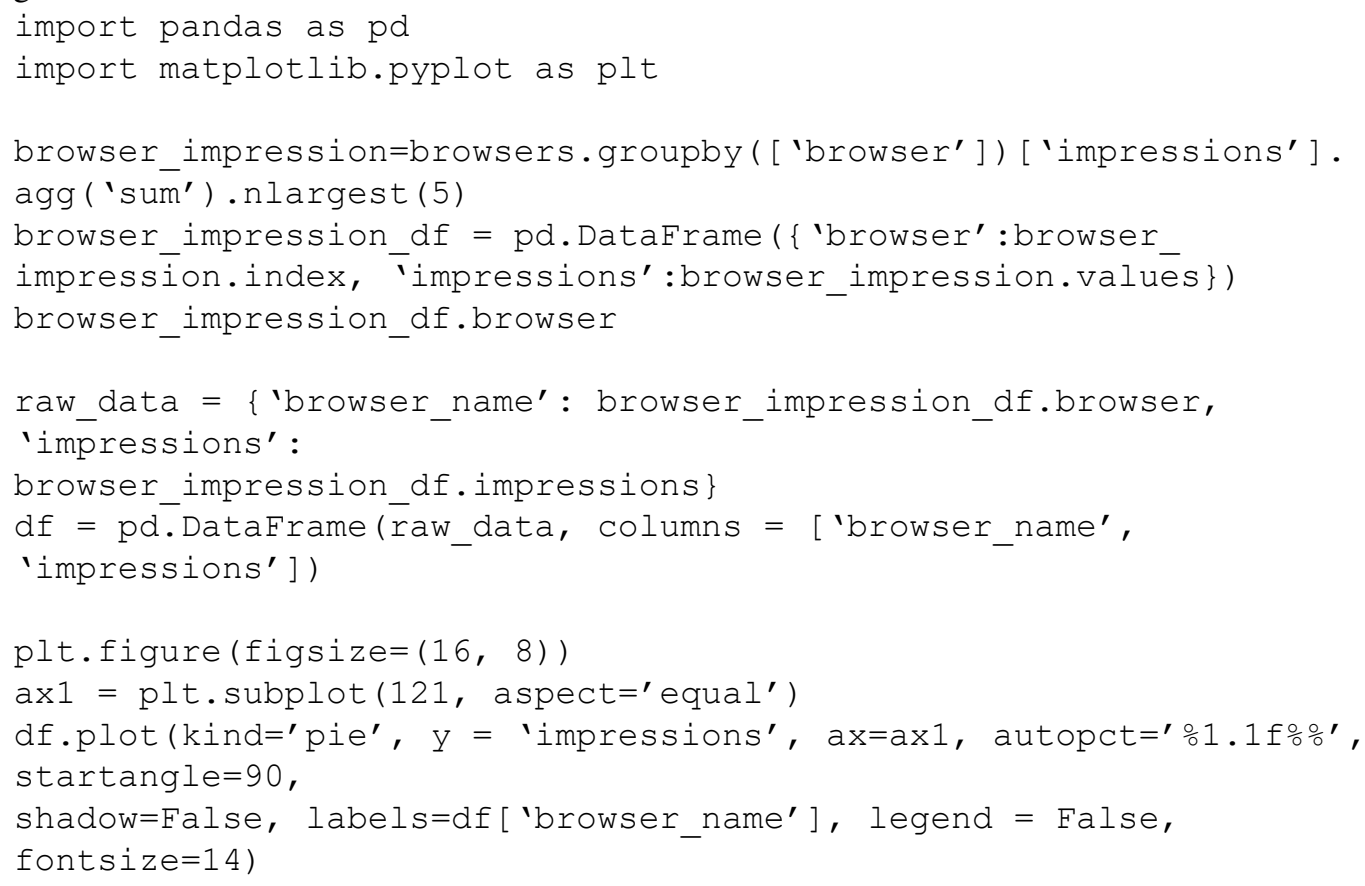

Finally, the authors looked at the placement category collection. This collection contains traffic details based on the placement category where the ad was shown. In this system, the authors found several categories such as entertainment, education, news, and unscored. Below is a block of code used to plot distribution of impressions per category. Also, the authors used Mongo database to find the sum of impressions per day and category. The query used for this calculation is given below. db.getcollection ("placement_categories") .aggregate (

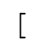




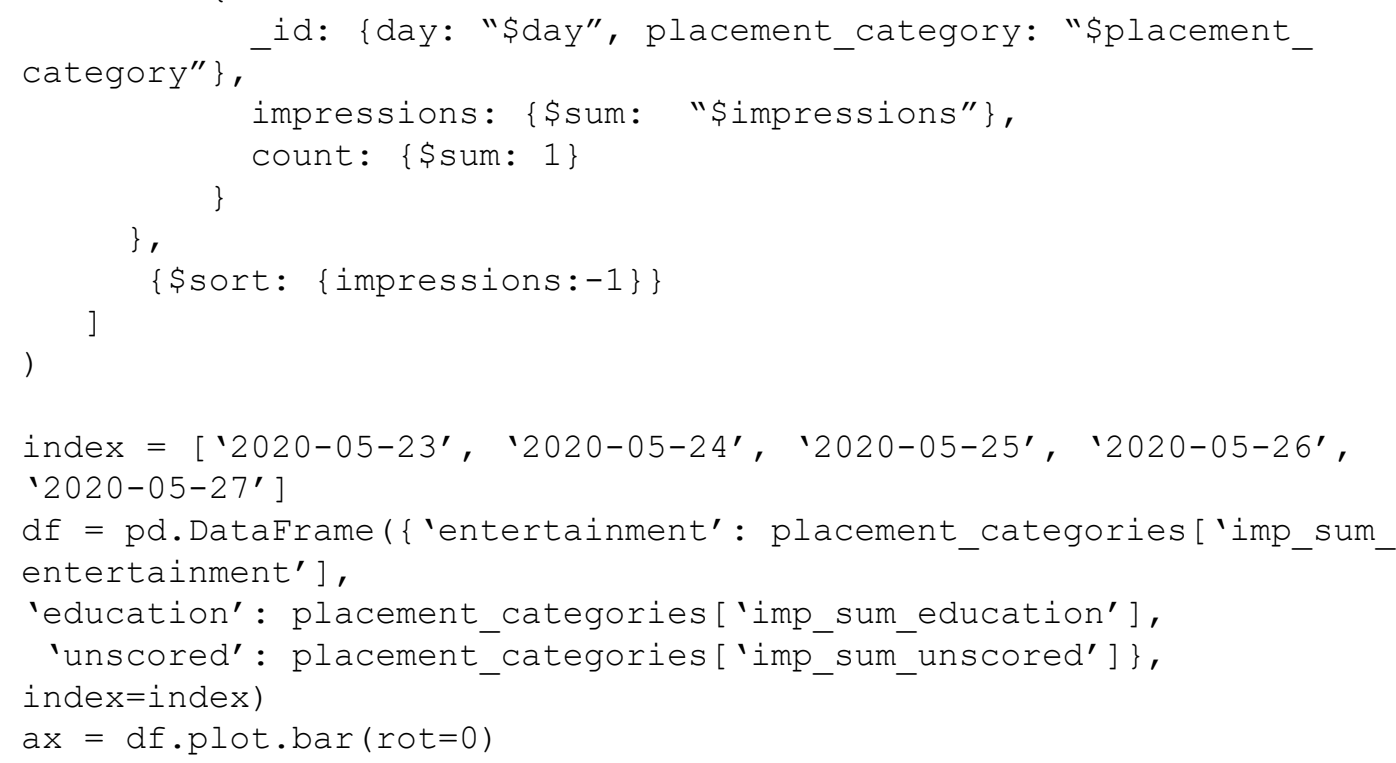

\section{Linear Regression}

In this study, simple linear regression was applied to understand the relationship between the number of impressions and clicks using scikit-learn (Scikit-Learn, n.d.) and statsmodels (Statsmodels, n.d.) Python libraries. The results could be affected by the difference in scale between the number of impressions and clicks because the number of clicks and sales in the traffic was very low, whereas the number of impressions was high.

For the purpose of building models, the authors used household income collection which filters traffic by household income categories such as $0-60 \mathrm{k}, 61-80 \mathrm{k}, 81-100 \mathrm{k}, 101-130 \mathrm{k}, 130+\mathrm{k}$. A block of code used to build the model, predict values, calculate p-value, and plot data is presented below.

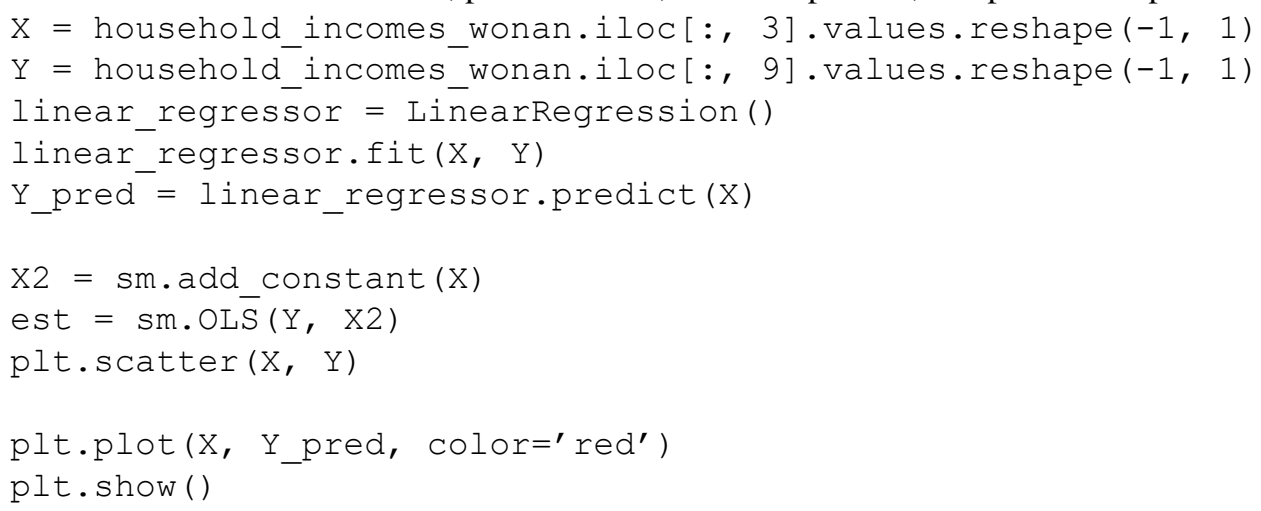

\section{RESULTS}

\section{Data Analysis}

Data analysis results show that 205 different countries generated traffic. Table 2 lists the top 10 countries that have the highest number of impressions, clicks, and sales. There are minor differences in the lists between impressions, clicks, and sales. For example, Taiwan, Israel, and Austria are not 
among the 10 countries with the highest number of impressions, but they are available in one of the two remaining lists.

Table 2. Ten countries with the highest number of impressions

\begin{tabular}{|l|l|l|l|}
\hline Country & Impressions & Clicks & Sales \\
\hline United States & 10429689 & $\mathbf{2 3 9 9}$ & $\mathbf{9 9}$ \\
\hline Canada & 100870 & 193 & 4 \\
\hline United Kingdom & 54729 & 76 & 4 \\
\hline China & 38060 & 43 & 10 \\
\hline Australia & 38004 & 27 & 2 \\
\hline Japan & 25847 & 28 & 5 \\
\hline Brazil & 17716 & & 3 \\
\hline France & 16410 & & 3 \\
\hline Germany & 15088 & 78 & 5 \\
\hline Republic of Korea & 12141 & 16 & \\
\hline Israel & & 1253 & \\
\hline Taiwan & & 16 & \\
\hline Austria & & & \\
\hline
\end{tabular}

Beside the results presented in Table 2, the authors also found that there are only 64 countries that generated click traffic. This means that 141 countries did not generate any clicks, but nevertheless, the budget was also spent on those countries.

Table 3 shows the distribution of traffic per day of the week. It is noticeable that Friday is the day with the highest number of impressions and sales, while the highest number of clicks is obtained on Monday. Bar charts for traffic per day are given in Figure 1, Figure 2, and Figure 3.

Figure 1. Distribution of impressions per days of the week

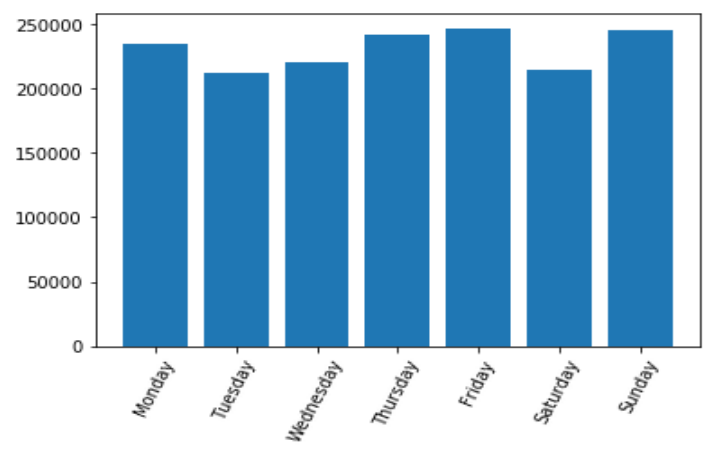


Figure 2. Distribution of clicks per days of the week

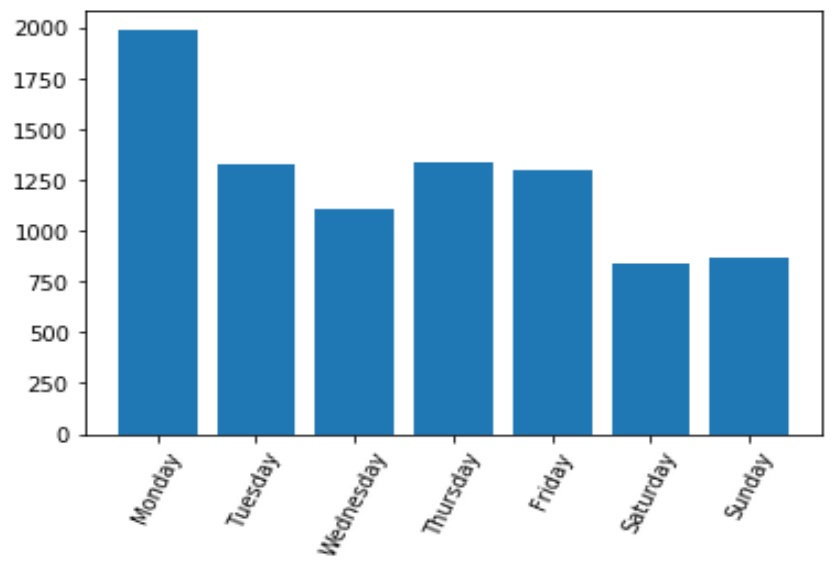

Figure 3. Distribution of sales per days of the week

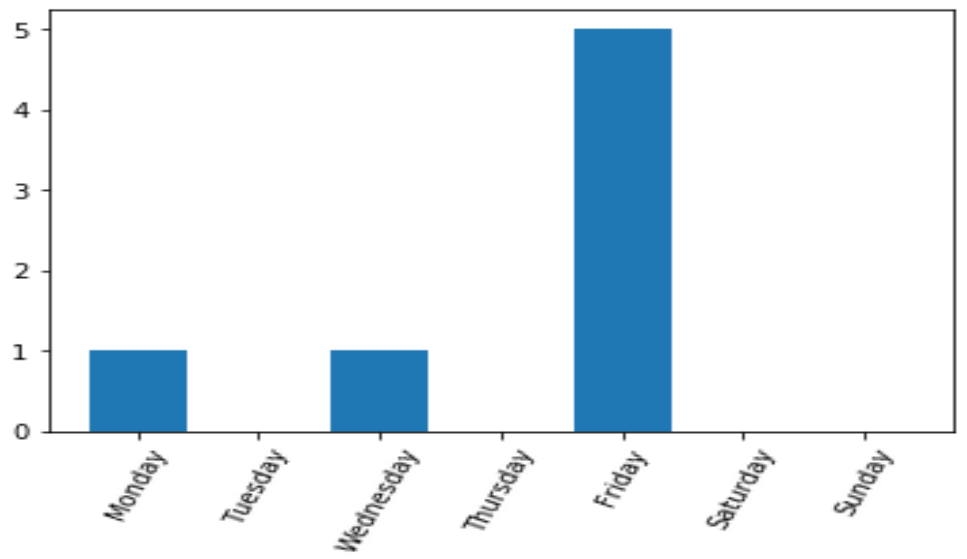

Table 3. Traffic distribution per day of the week

\begin{tabular}{|l|l|l|l|}
\hline Day of the week & Impressions & Clicks & Sales \\
\hline Monday & 234861 & $\mathbf{1 9 8 5}$ & 1 \\
\hline Tuesday & 212440 & 1325 & 0 \\
\hline Wednesday & 220196 & 1106 & 1 \\
\hline Thursday & 242216 & 1336 & 0 \\
\hline Friday & $\mathbf{2 4 6 3 1 8}$ & 1296 & $\mathbf{5}$ \\
\hline Saturday & 214345 & 838 & 0 \\
\hline Sunday & 245903 & 868 & 0 \\
\hline
\end{tabular}


Table 4. Top five browsers with the highest number of impressions, clicks, and sales

\begin{tabular}{|l|l|l|l|}
\hline Browser & Impressions & Clicks & Sales \\
\hline Chrome & $\mathbf{3 0 4 4 5 2 9}$ & $\mathbf{9 9 3 7}$ & 22 \\
\hline Safari & 2452712 & 3026 & 8 \\
\hline Android Webview & 910085 & 5412 & $\mathbf{1 2 1}$ \\
\hline Facebook Browser & 845192 & 767 & \\
\hline Mobile Safari UIWebView & 572196 & & 33 \\
\hline Edge & & 670 & \\
\hline Default Browser & & & 2 \\
\hline
\end{tabular}

Figure 4. Distribution of impressions per browser

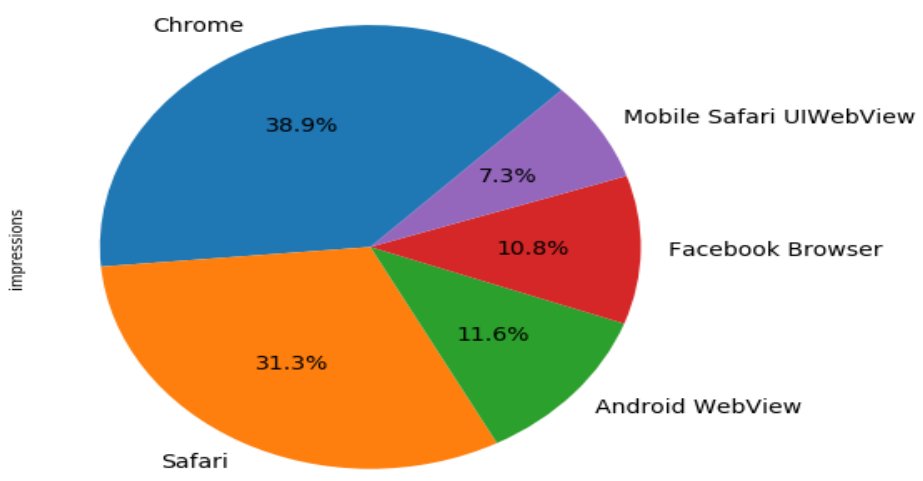

Figure 5. Distribution of clicks per browser

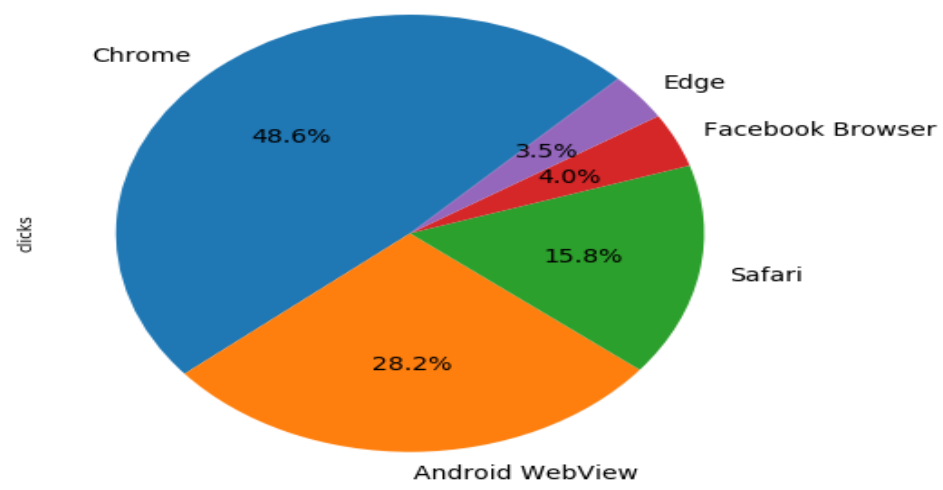




\section{Figure 6. Distribution of sales per browser}

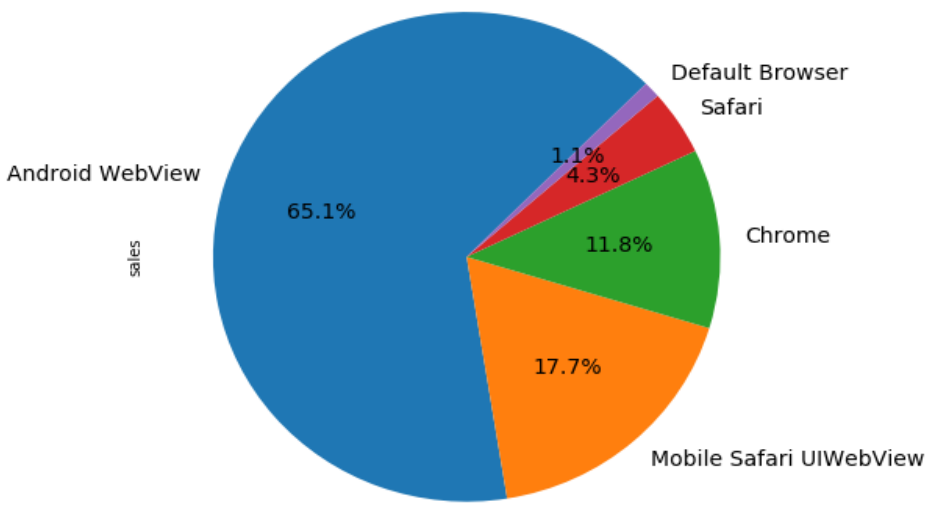

Figure 7. Distribution of impressions by categories per day

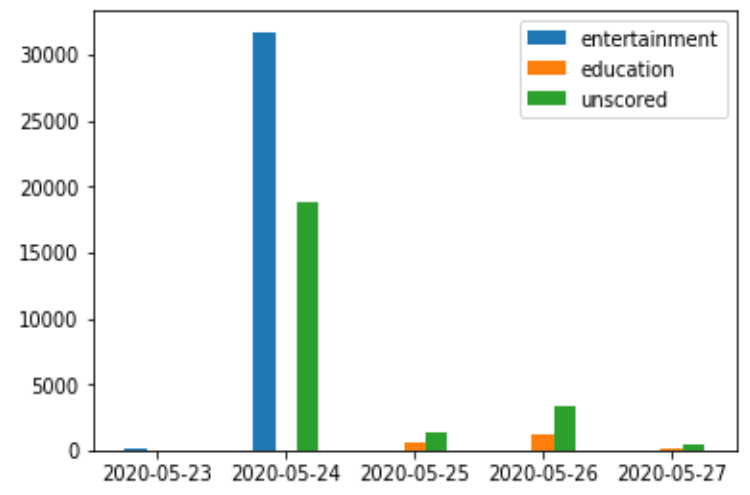

There is traffic detected for 278 different browsers. Table 4 lists 5 browsers with the highest number of impressions, clicks, and sales. Chrome, Safari, and Android Webview are browsers listed in the top 5 in all three categories. Results are visualized in the form of pie charts in Figure 4, Figure 5, and Figure 6 provided in the Appendix.

Figure 7 shows the distribution of impressions based on placement categories per day. Looking into the number of impressions, clicks, and sales, the authors noticed that a lot of traffic is recognized as unscored, which is a category that has not been determined yet. This means that this part of the data should be updated to categorize placements that are labeled as unscored. Moreover, it is necessary to expand the list of categories. Figure 7 shows that for 2020-05-24 the most of the impressions are obtained from the placement categorized as entertainment and those that don't have a category, respectively. Impressions for the rest of the presented days are obtained for placements that are not categorized and those recognized as education, respectively. 
Presented tables and figures can be used to build a dashboard that can be used to follow what is happening with the campaigns. Based on the results presented in this section, we could conclude that countries like the United States, Canada, and China should be targeted for advertising. This automatically excludes other countries that have low or no traffic. It can be concluded that Friday is the best day to increase the budget to collect impressions and sales. Most of the clicks are collected on Monday. Also, there is a list of browsers through which the most traffic is collected. This means that those browsers should be targeted, or at least the budget should be increased for those browsers and decreased for others that do not bring a lot of traffic.

\section{Linear Regression Model}

Figure 8 presents the results of linear regression. Although not highly emphasized, it could be noted that it is a positive regression, meaning that the number of clicks rises when the number of impressions is increased. Also, the obtained p-value was 0.5 .

Figure 8. Linear regression model

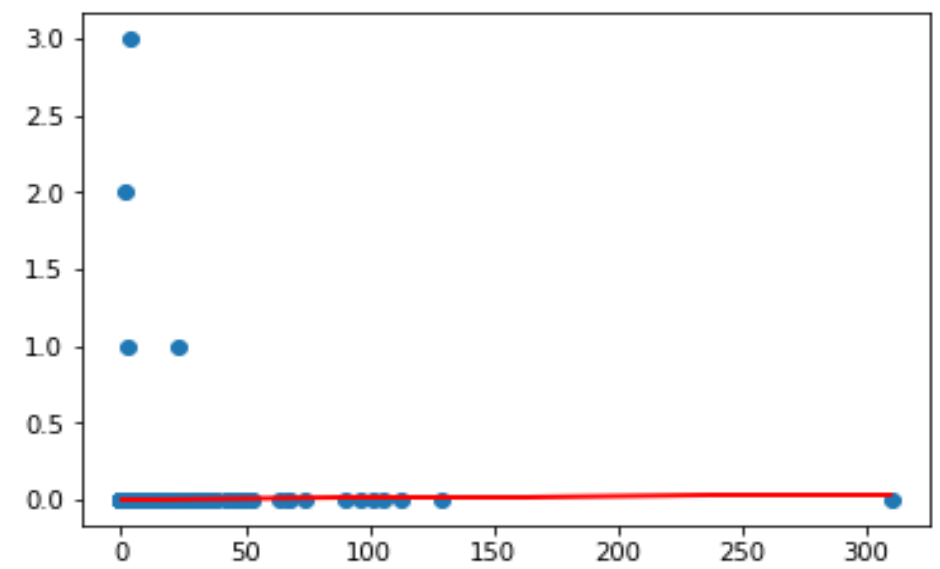

This kind of analysis can also be applied to find relationships between values and make conclusions about traffic and following steps.

\section{Proposed Reports}

By analyzing the dataset and different traffic, the authors came to numerous different report types that can be used to track traffic. Reports can be created for each traffic type. Reports can be generated for browsers, device types, brands and models, operating systems, locations, and others. These reports are generated for a date range, and for a more detailed view it is possible to provide details grouped by specific advertiser and campaign.

Alongside reports by traffic type, we can create reports based on the metric type, which are reports by impressions, clicks, or sales. Again, these reports can be given for a specific advertiser or campaign or as a general overview of the system. Since these reports are not based on traffic type, it could be useful to provide filters based on fields generated in the report. For our datasets, those could be country, region, city, zip code, browser, operating system, device type, mobile device brand and model. 
Beside these filters, it can be useful to capture the ad type or size and generate reports based on that. Furthermore, these reports can be generated on a daily or hourly basis to provide detailed insight into the system and campaign performance.

\section{CONCLUSION}

Data is a very important part of every business, but data alone is not enough. We must analyze data, find patterns, and make decisions based on analysis outcomes. In this paper, the authors presented the connection between business intelligence and digital advertising. The goal was to show that business intelligence can be applied to data collected from campaign traffic to evaluate campaign performance. Different data traffics based on targeting groups were examined.

The charts presented in the paper make it possible to identify the most useful targeting options in digital advertising. Chrome, Safari, and Android WebView are listed as the top browsers in all three measures: impressions, clicks, and sales. This means that those browsers should be used as target browsers.

Furthermore, the authors showed an example of how to use simple regression to find relationships between variables available in the system. Two variables were used, but multiple regression models could be applied to model a more complex system as well. This is important as a prediction tool to understand the system and enable smart decision-making according to the presented results.

Finally, several different kinds of reports were presented that should be generated and tracked to become aware of what is happening with campaigns. These reports can be used to make decisions regarding targeting optimization. Also, reports like these can be helpful in decision-making related to the budget for specific campaigns. Using such reports, a person who needs to make decisions can decide which targeting options should be included and which should be excluded. Moreover, results can be used to adjust budget options and increase return on investment. For example, one could increase the budget for a specific browser if it is found in reports that it brings more benefits than others. Also, some days or time of day could have a higher traffic rate, so it is recommended to spend more budget on these days or specific periods of a day and to save money or use it more wisely.

The findings in this study indicate that business intelligence can significantly help in decisionmaking and improve campaign performance in several ways. It allows tracking and analyzing generated traffic and make decisions based on those results. Following the results presented in this paper, we can conclude that countries like the United States of America, Canada, China, Japan, and Germany generate the majority of clicks and sales. This is highly significant because we can adjust targeting to include only those countries and use resources more adequately.

The results of the conducted research are considered as a contribution to the managerial activities by guiding the managers through understanding the role of the Web in the communication and sales channels. Also, segmentation methods are recommended to be used to understand the audience and define their behavior and habits. Furthermore, research and web analytics should be used to build successful marketing strategies.

The obtained reports are limited by the absence of behavioral data that would give a deeper insight into the audience's behavior. Also, it is considered that information about ad type would improve the results. A direction for future research is to analyze other traffic data such as keywords and topics. The analysis of the keywords and topics traffic could be used to split users and define different behavioral groups to build a more successful marketing strategy. 


\section{REFERENCES}

Bhosale, S. S., Sharma, Y. K., Kurupkar, F., \& Jhabarmal, S. J. (2020). Role of business intelligence in digital marketing. International Journal of Advance \& Innovative Research, 7(1).

Chaudhuri, S., \& Dayal, U. (1997). An overview of data warehousing and OLAP technology. SIGMOD Record, 26(1), 65-74. doi:10.1145/248603.248616

Dautovic, G. (2019, July 31). The 45 Most Important Advertising. SmallBizGenius. Retrieved from https://www. smallbizgenius.net/by-the-numbers/advertising-statistics/

Ecommerce Answers. (2020). Understanding Impressions in digital marketing. Retrieved August 30, 2020, from https://www.bigcommerce.com/ecommerce-answers/impressions-digital-marketing/

Ecommerce Answers. (2020). What is Cost-Per-Click (CPC) for ecommerce advertising? Retrieved August 30, 2020, from https://www.bigcommerce.com/ecommerce-answers/what-is-cost-per-click-cpc-for-ecommerceadvertising/

Folium. (n.d.). Retrieved September 4, 2020, from https://pypi.org/project/folium/

Frankenfield, J. (2019, June 23). Business Intelligence - BI. Guide to Mergers and Acquisitions. Retrieved from https://www.investopedia.com/terms/b/business-intelligence-bi.asp

Fuxman, L., Hilmi Elifoglu, I., Chao, C.-N., \& Li, T. (2018). Digital Advertising: A More Effective Way to Promote Businesses' Products. Journal of Business Administration Research, 3(2). https://doi.org/10.5430/jbar.v3n2p59

Greenspan, G. (2018). Digital Advertising. From Code to Product. Retrieved August 30, 2020, from http://www. gidgreen.com/course/2014-5-slides/06-digital-advertising.pdf

Imanuel. (2020, June 2). What is Data Analysis? Retrieved from https://www.predictiveanalyticstoday.com/ data-analysis/

Jalloh, A. A. A., \& Ming, F. (2020). Determinants of Employees Outstanding Performance in Public Corporations: Strategic Human Resource Management Perspectives of Organisational Development and Sustainability. International Journal of Management and Humanities, 5(1), 30-40. https://doi.org/10.35940/ijmh.a1 125.095120

Kline, J., Barford, P., Cahn, A., \& Sommers, J. (2017). On the structure and characteristics of user agent string. Proceedings of the 2017 Internet Measurement Conference, 184-190. 10.1145/3131365.3131406

Kurnia, P. F., \& Suharjito. (2018). Business Intelligence Model to Analyze Social Media Information. Procedia Computer Science, 135, 5-14. 10.1016/j.procs.2018.08.144

Kurniawan, Y., Gunawan, A., \& Kurnia, S. G. (2014). Application of business intelligence to support marketing strategies: A case study approach. Journal of Theoretical and Applied Information Technology, 64(1), 240-248.

Kursan, I., \& Mihić, M. (2010). Business Intelligence: The Role of the Internet in Marketing Research and Business Decision-Making. Management, 15, 69-86.

Matplotlib. (n.d.). Retrieved September 4, 2020, from https://pypi.org/project/matplotlib/

Nagpal, A., \& Gabrani, G. (2019). Python for Data Analytics, Scientific and Technical Applications. In 2019 Amity International Conference on Artificial Intelligence (AICAI). IEEE. https://doi.org/10.1109/aicai.2019.8701341.

Nichols, W. (2013, March 1). Advertising Analytics 2.0. Magazine. Retrieved from https://hbr.org/2013/03/ advertising-analytics-20

Pandas. (n.d.). Retrieved September 4, 2020, from https://pandas.pydata.org/

Scikit-learn. (n.d.). Retrieved September 6, 2020, from https://scikit-learn.org/stable/

Sharda, R., Turban, E., \& Delen, D. (2014). Business Intelligence and Analytics: Systems for Decision Support, Global Edition. Pearson.

Singh, A., \& Sharma, P. (2017). Comparative Analysis of MySQL (Relational) with MongoDB (NoSQL) Databases. Independently published. 
Statistics Solutions. (n.d.). What is Linear Regression? Retrieved August 30, 2020, from https://www. statisticssolutions.com/what-is-linear-regression/

Statsmodels. (n.d.). Retrieved September 6, 2020, from https://pypi.org/project/statsmodels/

Tableau. (2020). What is business intelligence? Your guide to BI and why it matters. Tableau Software. Retrieved July 6, 2020, from https://www.tableau.com/learn/articles/business-intelligence

Tilak, G. (2020). A Review of Using Business Intelligence (BI) in Digital Marketing. Studies in Indian Place Names (UGC Care Journal), 40(50).

tribeOS.io. (2020). Retrieved October 4, 2020, from https://tribeos.io/

Verma, S. (2018). Understanding the Role of Business Intelligence in Digital Marketing. International Journal of Scientific and Research Publications, 8(12), 17-19. https://doi.org/10.29322/ijsrp.8.12.2018.p8404

Waldron, M. W., Vsanthakumar, J., \& Arulraj, S. (1997). Improving the organization and management of extension. In B. E. Swanson, R. P. Bentz, \& A. J. Sofranko (Eds.), Improving agricultural extension. A reference manual. Food and Agriculture Organization of the United Nations.

WIRED Staff. (2010, October 27). Oct. 27, 1994: Web Gives Birth to Banner Ads. WIRED. Retrieved from https://www.wired.com/2010/10/1027hotwired-banner-ads/

Yale. (n.d.). Linear Regression. Retrieved August 30, 2020, from http://www.stat.yale.edu/Courses/1997-98/101/ linreg.htm

Yesbeck, J. (2018, February 8). The Importance of Targeting in Marketing (And How to Include It in Your Strategy). Retrieved from https://blog.alexa.com/targeting-in-marketing/

Zamfir, M., Manea, M. D., \& Ionescu, L. (2016). Return on Investment - Indicator for Measuring the Profitability of Invested Capital. Valahian Journal of Economic Studies, 7(2), 79-86. 10.1515/vjes-2016-0010

Zhang, K., \& Katona, Z. (2012). Contextual Advertising. Marketing Science, 31(6), 980-994.

Zhang, Y. (2015). An Introduction to Python and Computer Programming. In An Introduction to Python and Computer Programming (pp. 1-11). Springer. https://doi.org/10.1007/978-981-287-609-6_1.

Dželila Mehanović is Teaching Assistant in IT department at the Faculty of Natural Sciences at International Burch University, Sarajevo. She earned her master degree in information technologies in June 2019. Machine Learning applications are focus of Dželila's research.

Nermina Durmić is Assistant Professor in IT department at the Faculty of Natural Sciences at International Burch University, Sarajevo. She earned her PhD degree in technical sciences in the field of information technologies in April 2014. Software development following Agile principles is in the focus of Nermina's ongoing research. 